\title{
Preliminary study of effect of surface texturing on hypodermic needles
}

\author{
Divyansh Patel*, V. K. Jain, J. Ramkumar and Ankit Shrivastava \\ Department of Mechanical Engineering,, \\ Indian Institute of Technology Kanpur, Kanpur -208016), India \\ *Email: divy@iitk.ac.in
}

Insertion force of a sharp and cylindrical object into a soft solid body also depends on micro-features present on its interacting surface. Micro-textured surface of a hypodermic needle with a dimple array increases the friction between the needle and phantom tissue due to stress concentrations near the edges [1]. Some attempts were made imitating mosquito's proboscis to produce painless needles consisting of jagged shape structures on the outer surface of the needles [2,3]. In this relation, the paper presents an experimental study of fabrication of micro-textures on a hypodermic needle, and the effect of geometry of the micro-textures on the insertion force. A novel electrochemical microtexturing process is developed for generation of micro-features such as micro-dimples, circular micro-grooves and parallel micro-channels on curved metallic surfaces. In this article, a study related to the force required by of micro-textured hypodermic needle for insertion into soft hydrogel (whose physical properties are near to human skin) has been reported. Normal force with micro-textured needle is recorded using a strain gauge type dynamometer and then compared with the force required by a normal reference needle to show the effect of surface microtexture on the required insertion force. Figure 1 shows relation between insertion force and penetration depth when un-textured needle is inserted. Insertion force increases with increase in depth of penetration and the peaks of higher forces is obtained due to rupturing of gel when needle moves inside.

Figure 1: Trend of insertion force when a plain hypodermic needle is injected into hydrogel (Left), and a $16 \mathrm{~mm}$ hypodermic needle (Right)

Micro-texture parameters such as features areal density, depth and width are varied to extract the significance of each parameter. In this experimental study, it is observed that micro-features developed on the needle increase insertion force and change the behaviour of rupturing of hydrogel as compared to the needle without textures. Micro-grooves around the periphery (see Figure 2) show minimum increment in insertion force compared to other microtextured needles. In addition, the trend of increasing insertion force on increasing depth of penetration is almost linear. Figure 2(a) shows micro-dimples generated on surface of needle, (b) shows Microgrooves on the periphery and (c) shows the graph relating depth of penetration with insertion force. Figure 2(c) shows that, in case of textured needles, number peaks is less and the peak force value is low.
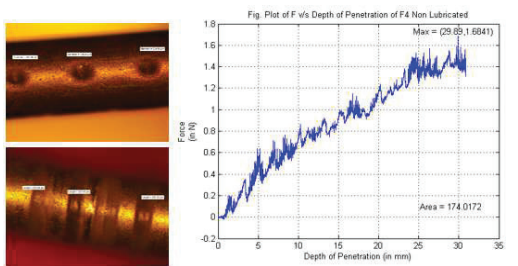

Figure 2: (a) Micro dimples array on hypodermic needles [4], (b) Micro-grooves array on the periphery of the hypodermic needle, (c) Graphs showing insertion force, when needle with micro-groves on its periphery is inserted into hydrogel

The paper concludes that the modification in geometrical parameters of micro-features to be generated on a hypodermic needle would decrease the pain to a patient. The idea of modification of the surface of the hypodermic needles which shall not only help eliminate the nightmares from the lives of many diabetic patients but also would help the governments round the globe in improving the coverage of their vaccination programs which is usually rendered ineffective due to a large needle phobic population. However, it has been identified that the technical difficulties underlay in the enhancement of the surface of such needles, which hinders the feasibility, and viability of the mass production of such needles.

\section{References}

1. X. Wang, M. Giovannini, Y. Xing, M. Kang, K. Ehmann, Tribol. Int. 92 (2015) 553-558.

2. H. Izumi, M. Suzuki, S. Aoyagi, T. Kanzaki, , Sensors Actuators, A Phys. 165 (2011) 115-123.

3. K. Oka, S. Aoyagi, Y. Arai, Y. Isono, G. Hashiguchi, H. Fujita, Sensors Actuators, A Phys. 97-98 (2002) 478-485. 4. D. Patel, V. K. Jain, Part B J. Eng. Manuf, (to be published). 